Received: 10 May 2018

Accepted: 25 October 2018

Published online: 22 January 2019

\section{No Association between staging operation and the 5-Year Risk of Reoperation in Patients with Crohn's Disease}

\author{
Jiajie Zhou ${ }^{1,2}, \mathrm{Yi} \mathrm{Li}^{1}$, Jianfeng Gong ${ }^{1}$ \& Weiming $\mathrm{Zhu}^{1}$
}

The aim of this study was to investigate the impact of staging operation on the risk of reoperation in patients with CD who underwent primary bowel resection. This was a retrospective study of 980 patients with CD who were hospitalized in Jinling Hospital Affiliated to Nanjing Medical University between January 1, 2001, and October 1, 2016. The patients were grouped according to staging operation $(n=64)$ and one-stage operation $(n=148)$. Postoperative intestinal function recovery time, postoperative short-term complications, and reoperation rates were compared between the two groups. There was significant difference in disease behavior between the staging operation group and the one-stage operation group. There was no significant difference in postoperative tolerance of enteral nutrition among groups $(P>0.05)$. Obvious differences were found in the comparison of the first time of exhaustion, defecation after operation, postoperative length of stay and postoperative complications among groups (all $\mathrm{P}<0.05$ ). There was no difference in the 5 -year cumulative reoperation-free rates between the two groups $(P>0.05)$. In conclusion, surgical intervention at proper time and appropriate operation during operation are essential for patients with CD. It is believed that staging operation with ostomy followed by intestinal anastomosis is feasible when there are more than two risk factors for postoperative intra-abdominal infectious complications.

Crohn's disease (CD) is a chronic inflammatory granulomatous disease of the intestines with lifelong relapse. Even though internal medicine is the major therapeutic approach, $70 \sim 90 \%$ of the disease requires surgical intervention eventually ${ }^{1-3}$. Surgical intervention is used frequently for intestinal stenosis, perforation, abdominal abscess and other complications caused by recurrent intestinal inflammation. CD patients required surgical treatment not only have CD complications that need to be managed by surgery, but also suffer from malnutrition caused by long-term intestinal dysfunction and immune disorders due to long-term administration of drugs. Some patients even require emergency surgery that significantly limit adequate perioperative preparation.

The principle of surgical treatment for CD is to control the symptoms, and corresponding operative modes include diseased intestinal resection, ostomy and drainage of abdominal abscess, etc. When the whole-body situation of the patient is not allowed to perform major incision, surgical treatment in accordance with the concept of "damage control surgery (DCS)" can reduce the risk of treatment to a certain extent and improve corresponding clinical effect. In 1990s, Rotondo ${ }^{4}$ formally put forward the concept of DCS, and expounded the three-stage principle of DCS, including fast control of the damage, resuscitation, and definitive operation. For critically ill patients with CD, under the guidance of the DCS concept, staging surgery is commonly adopted, with the performance of one-stage ostomy to deal with intestinal complications possibly, and restore intestinal function rapidly, so as to improve the nutritional status of the patients. Furthermore, definitive operation will be carried out on the basis of general condition improvement in the patients. Staging operation is a common approach for CD patients. Nevertheless, there is no relevant literature so far to verify whether the risk of reoperation in these patients is increased with staging operation compared with one-stage definitive operation.

${ }^{1}$ Department of General Surgery, Jinling Clinical Medical College, Nanjing Medical University, Nanjing, Jiangsu, 210002, China. 'Department of General Surgery, Huai'an First People's Hospital, Nanjing Medical University, Huai'an, Jiangsu, 223300, China. Correspondence and requests for materials should be addressed to W.Z. (email: juwiming@ nju.edu.cn) 


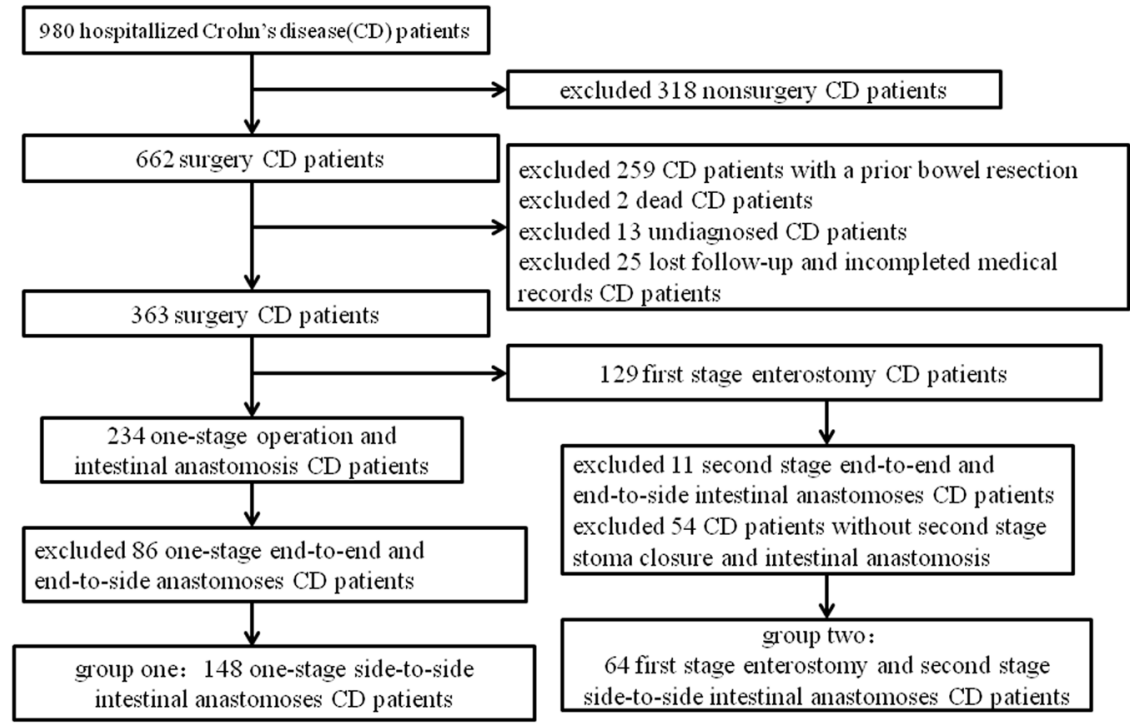

Figure 1. Study selection flow chart.

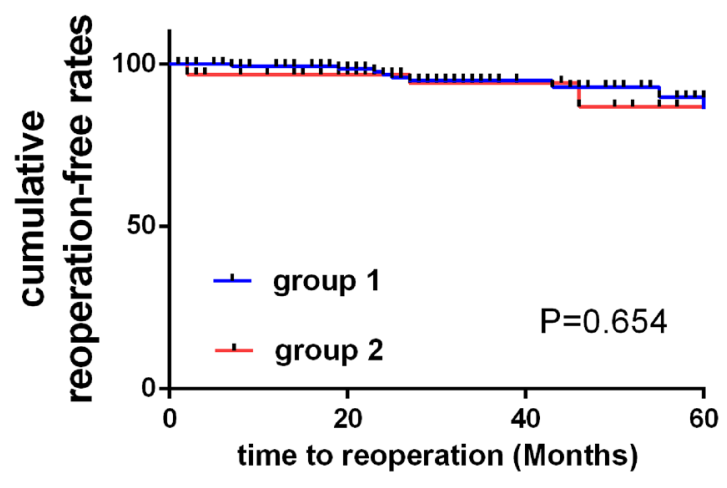

Figure 2. Kaplan-Meier curves of cumulative reoperation-free rates in the two groups are shown in Fig. 2.

Therefore, the present study was conducted to explore the effect of staging operation on postoperative reoperation rate of $\mathrm{CD}$ patients. The results of this study may be beneficial for optimizing the therapeutic regimen and improve the quality of life in CD patients postoperatively.

\section{Materials and Methods}

Study Design and Patients. This study was approved by the ethics committee of Jinling Hospital Affiliated to Nanjing Medical University. The need for individual consent was waived by the committee because of the retrospective nature of the study. This was a retrospective study of 980 patients with CD who were hospitalized in Jinling Hospital Affiliated to Nanjing Medical University between January 1, 2001, and October 1, 2016.

Inclusion criteria of eligible patients: (1) The diagnosis and treatment of CD were carried out based on the European Crohn's and Colitis Organization (ECCO) consensus standard ${ }^{5,6}$; (2) CD patients with one-stage resection and intestinal anastomosis for the first time in our hospital; (3) Patients with staging operation who underwent ostomy in our hospital for the first time, as well as two-stage ostomy and intestinal anastomosis. The exclusion criteria were: (1) incomplete medical records; or (2) Patients who had enteric resection and enterostomy in other hospital prior to admission. The diagnosis and treatment of all patients were accordant with the recommended standard of ECCO consensus.

The strategy used on selected patients was shown in Fig. 1. A total of $148 \mathrm{CD}$ patients underwent one-stage stapled side-to-side anastomosis (group one) and $64 \mathrm{CD}$ patients with first stage enterostomy and second stage side-to-side anastomoses (group two) were included in the final analysis.

Group one patients underwent primary intestinal resection with one-stage stapled side-to-side anastomosis. cover stoma was not allowed on this group. All Group two patients received two-stage operation. A temporary stoma might be adopted for these patients with emergency visit, poor nutritional status or risk factors for postoperative abdominal infection at first stage. Besides, during the first stage operation, intestinal lesions would be treated simultaneously or with the performance of exclusion surgery at the same time, and cover the stoma at second stage operation with or without definitive operation. CD patients with permanent stoma after the 
first stage operation were excluded from the study. Only disease recurrence related operations were considered reoperations.

Indications for two-stage operations included acute diffuse peritonitis and two or more risk factors. Risk factors for postoperative abdominal infection included poor preoperative nutritional status (preoperative albumin levels $<35 \mathrm{~g} / \mathrm{l}$ ), preoperative hormone use, preoperative intraperitoneal abscess, preoperative immunosuppressive agents and biological agents.

Group one patients and Group two patients before second stage operation needed to improve nutritional status (preoperative albumin levels $>35 \mathrm{~g} / \mathrm{L}$ ) and induce disease remission (c-reactive protein $<8 \mathrm{mg} / \mathrm{L}$ ) preoperation by enteral nutrition, abdominal abscess drainage and anti-infection treatment.

Pre- and Perioperative Management. All patients underwent perioperative management according to the enhanced recovery after surgery model (ERAS $)^{7,8}$, including no mechanical bowel preparation, drinking water $2 \mathrm{~h}$ before surgery, having solid diet $6 \mathrm{~h}$ before surgery, preoperative non-prophylactic use of intestinal antibiotics, intraoperative limiting of fluid intake (less than $1500 \mathrm{ml}$ ), postoperative non-routine gastric tube implantation, early pulling out urinary catheter, early oral diet and out of bed activity, prohibition of opioid analgesics, and postoperative limited fluid therapy (according to blood lactate level). In group one, 63 patients were treated with azathioprine preoperation. 6 patients received anti-TNF drugs before operation. In group two, 41 patients were treated with azathioprine before second stage operation. 3 patients received anti-TNF drugs before second stage operation.

Postoperative Management. All patients were treated with enteral nutrition early in the postoperative period, and administrated with azathioprine $(1.5 \mathrm{mg} / \mathrm{kg} / \mathrm{d})$ orally within two weeks after operation. Medication would be replaced if the patients could not tolerate azathioprine. Detailed information related to postoperative medication was described in Table 1.

Data Collection. The clinical data were collected from the hospital database, including the patient's general situation, preoperative and postoperative medication, surgical records, Montreal classification, and reoperation. The outpatient and inpatient records of all CD patients were retrospectively analyzed. As per out institution's standard, all patients with CD are followed up at the outpatient clinic after surgery. If follow-up made at another hospital or if a visit is skipped, telephone follow-up is done. Patients who were nonetheless lost to follow-up were censored at their last visit.

Montreal typing ${ }^{9}$ was used to describe the patient's age at diagnosis, location of the disease, and disease behavior. According to the age of diagnosis, patients were divided into three grades: (A1) < 16 years old; (A2) 17-40 years old; and (A3) > 40 years old. Patients were classified into: (L1) ileum; (L2) colon; (L3) colon; and (L4) upper alimentary tract based on the location of the disease. In accordance with disease behavior, patients were divided into: (B1) non-stenotic and non-penetrating lesions; (B2) stenotic lesions; (B3) penetrating lesions; and (P) perianal lesions.

The staging operation referred to an operation plan that a temporary ostomy might be firstly adopted for patients with emergency visit, poor nutritional status or risk factors for postoperative abdominal infection. Besides, during the operation, intestinal lesion would be treated simultaneously or with the performance of exclusion surgery at the same time, and definitive operation would be performed during two-stage ostomy and intestinal anastomosis. In addition, the one-stage operation was defined as the one-stage resection of the intestinal canal and the side-to-side anastomosis of the intestine following adequate perioperative preparation, improvement of nutritional status in patients, and normal preoperative maintenance of inflammation indexes.

Outcomes. To group one (one-stage operation) CD patients, follow-up started on the initial operation date. To group two (staging operation) CD patients, follow-up started on the second stage operation date. The primary study outcome was reoperation during follow-up. The disease duration was defined as the time from diagnosis to initial surgery. Perioperative complications were observed according to the Dindo-Clavien classification criteria ${ }^{10}$ : grade I: without requirement of other treatment in addition to the antiemetic, antipyretic, and analgesic agents, diuretic, and electrolyte, physiotherapy, or bedside opening of infected wounds; grade II: need for medical treatment, antibiotic treatment for incision infection, or need for blood transfusions and total parenteral nutrition; grade III: need for surgical, endoscopic, or interventional radiology treatment; grade IV: requirement of intermittent monitoring or transfer to the ICU for life-threatening complications (including central nervous system complications); and grade V: death.

Statistical Analysis. All data were analyzed using SPSS 21.0 (IBM, Armonk, NY, USA). Continuous data distribution was determined using the Kolmogorov-Smirnov test. Normally distributed data were presented as mean \pm standard deviation and analyzed using the Student $t$ test. Non-normally distributed data were presented as median (range) and analyzed using the Mann-Whitney $U$ test. Categorical data were presented as frequencies and analyzed using the chi-square or Fisher exact test, as appropriate. The cumulative reoperation rate was calculated using the Kaplan-Meier method and analyzed using the log-rank test. Two-sided P-values $<0.05$ were considered statistically significant.

\section{Results}

Characteristics of the Patients. As shown in Table 1, there was no difference in terms of gender, course of disease, preoperative history of operation, age of diagnosis, location of disease and medication after operation between groups. Furthermore, obvious differences were observe regarding operation mode, operation timing and disease behavior between groups. Meanwhile, rates for laparoscopic operation and elective surgery were higher in the one-stage operation group than those in the staging operation group, while the proportion of penetrating 


\begin{tabular}{|c|c|c|c|}
\hline Variables & $\begin{array}{l}\text { Group } 1 \\
(n=148)\end{array}$ & $\begin{array}{l}\text { Group } 2 \\
(n=64) \text { first stage }\end{array}$ & $\mathbf{P}$ \\
\hline Female & $91(61.5 \%)$ & $49(76.6 \%)$ & 0.591 \\
\hline Duration of disease (months) & $35(1-226)$ & $33(1-243)$ & 0.579 \\
\hline \multicolumn{4}{|l|}{ Prior surgery history } \\
\hline Abdominal abscess drainage & $35(23.6 \%)$ & $10(61.5 \%)$ & 0.190 \\
\hline Appendectomy & $28(18.9 \%)$ & $12(15.6 \%)$ & 0.977 \\
\hline Perianal operation & $26(17.6 \%)$ & $14(21.9 \%)$ & 0.462 \\
\hline Smoking history & $9(6.1 \%)$ & $5(7.8 \%)$ & 0.641 \\
\hline \multicolumn{4}{|l|}{ Surgical approach } \\
\hline Laparoscopic/open & $58 / 90$ & $8 / 56$ & $<0.001$ \\
\hline \multicolumn{4}{|l|}{ Timing of operation } \\
\hline emergency/selective & $1 / 147$ & $9 / 55$ & $<0.001$ \\
\hline \multicolumn{4}{|c|}{ Montreal Classification at diagnosis } \\
\hline Age of diagnosis & & & 0.713 \\
\hline A1 ( $\leq 16$ years) & $11(7.4 \%)$ & $8(12.5 \%)$ & \\
\hline A2 (17-40 years) & $107(72.3 \%)$ & $42(65.6 \%)$ & \\
\hline A3 ( $>40$ years) & $30(20.3 \%)$ & $14(21.9 \%)$ & \\
\hline Location of disease & & & 00.363 \\
\hline L1 (Terminal ileum) & $69(46.6 \%)$ & $26(40.6 \%)$ & \\
\hline L2 (Colonic) & $9(6.1 \%)$ & $13(20.3 \%)$ & \\
\hline L3 (Ileocolonic) & $44(29.7 \%)$ & $24(37.5 \%)$ & \\
\hline L4 (Upper GI) & $26(17.6 \%)$ & $1(1.6 \%)$ & \\
\hline Behavior of disease & & & $<0.001$ \\
\hline B1 (Non-str/non-pen) & $8(5.4 \%)$ & $5(7.8 \%)$ & \\
\hline B2 (Stricturing) & $85(57.4 \%)$ & $16(25 \%)$ & \\
\hline B3 (Penetrating) & $55(33.5 \%)$ & $43(67.1 \%)$ & \\
\hline $\mathrm{P}$ (Perianal lesions) & $34(29.8 \%)$ & $20(31.2 \%)$ & 0.204 \\
\hline Postoperative management & & & 0.253 \\
\hline Mesalazine & $13(8.8)$ & $2(3.1 \%)$ & \\
\hline Azathioprine & $115(77.7 \%)$ & $48(75 \%)$ & \\
\hline Remicade & $2(1.4 \%)$ & $2(3.1 \%)$ & \\
\hline Tripterygium glycosides & $17(11.5 \%)$ & $10(15.6 \%)$ & \\
\hline Thalidomide & $1(0.7 \%)$ & $2(3.1 \%)$ & \\
\hline
\end{tabular}

Table 1. Characteristics of the Patients. GI: gastointestinal; non-str: non-stricturing; non-pen: non-penetrating. Group A: one-stage operation group; Group B: staging operation group.

lesions was lower than that in the latter group. However, the proportion of stenotic lesions was higher in the one-stage operation group than that of the staging operation group. The median time of ostomy and intestinal anastomosis was 7 months (3-24) in the staging operation group.

Comparison of Postoperative Observation Indexes between Groups. As illustrated in Table 2, there was no significant difference in postoperative tolerance of enteral nutrition among groups $(P>0.05)$. Obvious differences were found in the comparison of the first time of exhaustion, defecation after operation, postoperative length of stay and postoperative complications among groups (all $\mathrm{P}<0.05$ ). Compared with patients in the one-stage operation group and patients with ostomy and intestinal anastomosis in the staging operation group, the postoperative exhaustion and defecation time were much earlier in patients with ostomy of the staging operation group. Nevertheless, the time of postoperative length of stay was longer in patients with ostomy of the staging operation group than those of the former two groups. Furthermore, in patients with ostomy of the staging operation group, postoperative grades II, III and IV complications were higher than those of the other two groups. Moreover, there was no IV complication in the latter two groups. After ostomy in the staging operation group, there were 5 cases of IV complication, 3 cases of septic shock, 1 case of anastomotic bleeding and 1 case of cardiac insufficiency, all of which were improved after supportive treatment when transferring to the ICU.

Comparison of Cumulative Reoperation-Free Rates. No patient died during the follow-up period. In group one (148 patients), 11 patients (7.43\%) needed reoperation due to disease recurrence after primary operation during the follow-up period. In group two (64 patients), 4 patients $(6.25 \%)$ as well as required reoperation because of disease recurrence after second stage operation during the follow-up period. The 5-year cumulative reoperation-free rate of the two groups was illustrated in Fig. 1. There was no statistically significant difference in reoperation-free rate between groups within 5 years $(\mathrm{P}=0.654)$. 


\begin{tabular}{|c|c|c|c|c|}
\hline \multirow[b]{2}{*}{ Postoperative observation indexes } & \multirow{2}{*}{$\begin{array}{l}\text { Group1 } \\
(n=148)\end{array}$} & \multirow{2}{*}{$\begin{array}{l}\begin{array}{l}\text { Group } 2 \\
(n=64)\end{array} \\
\text { first stage }\end{array}$} & \multirow{2}{*}{\begin{tabular}{|l|}
$\begin{array}{l}\text { Group 2 } \\
(\mathrm{n}=64)\end{array}$ \\
second stage \\
\end{tabular}} & \multirow[b]{2}{*}{$\mathbf{P}$} \\
\hline & & & & \\
\hline Time of exhaustion (days) & $3(1-8)$ & $2(1-13)$ & $3(2-7)$ & $<0.001$ \\
\hline Time of defecation (days) & $3(1-9)$ & $2(1-13)$ & $3(2-7)$ & $<0.001$ \\
\hline Tolerance period of enteral nutrition (days) & $4(2-20)$ & $4(1-15)$ & $4(2-10)$ & 0.653 \\
\hline Postoperative hospital stay (days) & 6(3-33) & $9(3-56)$ & $7.5(3-25)$ & 0.021 \\
\hline Postoperative complications & & & & 0.013 \\
\hline Grade I & $20(13.5 \%)$ & $6(9.3 \%)$ & $12(18.8 \%)$ & \\
\hline Abdominal distension & $4(2.7 \%)$ & $2(3.1 \%)$ & $2(3.1 \%)$ & \\
\hline Infection of incisional wound & $8(5.4 \%)$ & $4(6.3 \%)$ & $9(14.1 \%)$ & \\
\hline Diarrhea & $7(4.7 \%)$ & & & \\
\hline Hydrothorax & $1(0.7 \%)$ & & $1(1.7 \%)$ & \\
\hline Grade II & $28(18.9 \%)$ & $24(37.5 \%)$ & $18(28.1 \%)$ & \\
\hline Intestinal obstruction & $1(0.7 \%)$ & $1(1.6 \%)$ & & \\
\hline Incision bleeding & $1(0.7 \%)$ & $1(1.6 \%)$ & & \\
\hline Fungal infection & & $1(1.6 \%)$ & & \\
\hline Abdominal distension & $12(8.1 \%)$ & $4(6.3 \%)$ & $11(17.2 \%)$ & \\
\hline High-output stoma & & $7(10.9 \%)$ & & \\
\hline Anemia & $6(4.1 \%)$ & $10(15.6 \%)$ & $1(1.6 \%)$ & \\
\hline Anastomotic bleeding & $1(0.7 \%)$ & & $1(1.6 \%)$ & \\
\hline Diarrhea & $5(3.4 \%)$ & & $5(7.8 \%)$ & \\
\hline Intra-abdominal infection & $1(0.7 \%)$ & & & \\
\hline Pulmonary infection & $1(0.7 \%)$ & & & \\
\hline Grade III & $9(6.1 \%)$ & $7(10.9 \%)$ & $4(6.3 \%)$ & \\
\hline Duodenal fistula & & $1(1.6 \%)$ & & \\
\hline Cholecystitis & & $1(1.6 \%)$ & & \\
\hline Pleural effusion & & $3(4.7 \%)$ & $1(1.6 \%)$ & \\
\hline Hydrops abdominis & $2(1.4 \%)$ & $2(3.1 \%)$ & & \\
\hline Anastomotic fistula & $4(2.7 \%)$ & & $1(1.6 \%)$ & \\
\hline Intra-abdominal hemorrhage & $1(0.7 \%)$ & & & \\
\hline Incision hemorrhage & $1(0.7 \%)$ & & $1(1.6 \%)$ & \\
\hline Intra-abdominal abscess & $1(0.7 \%)$ & & $1(1.6 \%)$ & \\
\hline Grade IV & & $5(7.8 \%)$ & & \\
\hline Infectious shock & & $3(4.7 \%)$ & & \\
\hline Anastomotic hemorrhage & & $1(1.6 \%)$ & & \\
\hline Cardiac failure & & $1(1.6 \%)$ & & \\
\hline
\end{tabular}

Table 2. Postoperative observation indexes.

\section{Discussion}

In this study, there was no difference in gender, course of disease, previous surgical history, age of diagnosis, location of the disease, and postoperative medication in the observed patients between groups. Moreover, significant differences were observed between groups regarding operation mode, operation timing and disease behavior. The above results might be attributed to the selection of treatment plan for CD patients in this IBD center. Intraperitoneal lesions was evaluated by hematopoietic, endoscopic and imaging examinations. For patients with one-stage operation of definitive operation, adequate perioperative management was carried out, including perioperative nutritional support and nutritional status improvement. Abdominal puncture and drainage were performed in patients with abdominal abscess. After preoperative reduction of inflammatory indexes levels in the blood to the normal range, the lesion was resected with one-stage anastomosis. Furthermore, for patients undergoing emergency operation and with poor nutritional status or severe intraoperative abdominal contamination, staging operation was adopted by using one-stage temporary ostomy and two-stage intestinal anastomosis. In addition, for patients who required temporary ostomy, abdominal abscess drainage or diseased intestinal resection would be performed during operation, and enteral nutrition support was given early after operation. Prior studies ${ }^{11}$ docu- $^{-}$ mented that perioperative nutritional support could not only reduce the incidence of postoperative complications, but also induce CD remission, eliminate intestinal obstruction, promote spontaneous closure of enterocutaneous fistula and avoid another risk of surgery. As proved in the study conducted by Gutierrez ${ }^{12}$, in CD patients required surgery, the abscess drainage should be performed firstly when combining with the formation of abscess, and some patients no longer needed surgical treatment after abscess dissipation. The first choice for abscess drainage was percutaneous puncture and drainage ${ }^{13}$. In such way, some perforated lesions of the intestines could be healed and the possibility of intestinal resection could be reduced accordingly ${ }^{14}$. Besides, the risk of operation could be significantly decreased simultaneously even if it was necessary to remove the penetrating ulcer. 
Previous evidence ${ }^{15}$ suggested that intestinal fistula, abdominal infection, malnutrition and other risk factors were potential risk factors for postoperative complication of abdominal infection. Intra-abdominal septic complications (IASC) (Anastomotic leakage, abdominal abscess, or peritonitis) were risk factors for postoperative recurrence of the disease ${ }^{16}$. A Study ${ }^{17}$ has shown that nearly $50 \%$ of patients required reoperation due to postoperative complications in patients with abdominal cavity infection. It was indicated that during staging operation, the application of temporary ostomy could improve the safety of operation and reduce the incidence of IASC remarkably ${ }^{15,17-19}$. At present, postoperative IASC risk factors are considered to include poor preoperative nutritional status, preoperative hormone use, preoperative intraperitoneal abscess, preoperative immunosuppressive agents and biological agents, etc ${ }^{15,16,19}$. Furthermore, as verified by past studies, with the increase of risk factors, the incidence of postoperative IASC would be increased at the same time. Moreover, the incidence of postoperative complications would elevate to $40 \%$ when there was preoperative hormone use history and abdominal abscess ${ }^{19}$. Therefore, during preoperative evaluation, it is recommended that staging operation with ostomy and intestinal anastomosis should be performed primarily if there are two or more risk factors in the target patients. The study ${ }^{17}$ has also discovered that the first implementation of ostomy could significantly reduce risk factors of the operation and incidence of postoperative complications, without clear impact on the overall number of operations and postoperative length of stay, which was consistent with the results of our study. In addition, over 75\% of patients could recover intestinal continuity ${ }^{20}$, besides, the median time of ostomy and intestinal anastomosis was about 5 months. In the present study, the median time of ostomy and intestinal anastomosis was estimated to be 7 months.

Our study found that Group two patients at the first stage operation had higher proportion of penetrating diseases and emergency surgery than one-stage operation patients. The literature ${ }^{5,6}$ shows that penetrating lesions and emergency surgery are risk factors for reoperation, but our study found no difference in long-term reoperation rates between one-stage and two-stage operation groups. We thought two-stage operation patients had poor nutritional status and disease active at their first stage operation. This situation led to their proportion and grade of postoperative complications were significantly higher than one-stage operation patients. Due to the first stage operation, their nutrition and infection status had been better at the second stage of opertion. When staging operation patients received the second stage operation, they were under the similar condition to surgery, resulting in no difference in long-term reoperation rates between two groups.

In the present study, the postoperative length of stay, as well as the proportion and level of postoperative complications were estimated to be higher for patients with ostomy in the staging operation group than those in the one-stage operation group. Possible reason might be attributed to the poor general condition and critically ill situation of patients who required ostomy. However, there was no difference in postoperative exhaustion and defecation, early postoperative enteral nutrition support, postoperative length of stay, and postoperative complications in patients undergoing ostomy and intestinal anastomosis in the staging operation group when compared to those in the one-stage operation group.

The advantages of ostomy were early postoperative exhaustion and defecation, rapid providing of enteral nutrition, and improvement in the nutritional status of patients, and there was no risk of anastomotic leakage. Existing literature ${ }^{21-23}$ showed that fecal passage diversion could significantly improve the inflammation of distal indwelling colon. The "sutures with mucosal eversion" technique ${ }^{24}$ greatly reduced the incidence of complications of ostomy in our study. Besides, no complications such as stomal stenosis, para-stomal hernia and stoma necrosis occurred after ostomy and during intestinal anastomosis. However, about $11 \%$ of patients underwent ostomy appeared water-electrolyte disturbance due to postoperative excessive loss of ostomy fluid, which were eventually improved after timely supportive treatment. Studies ${ }^{25}$ have evaluated that the incidence of water-electrolyte disturbance was the highest in CD patients after ostomy, accounting for $29 \%$ of all complications. Besides, loop ileostomy was the main risk factor for patients with terminal ileostomy. In those patients ${ }^{26}$, the intake and discharge of liquid should be monitored closely, in combination with the restriction of the intake of hypotonic solution. If necessary, isotonic liquid, antidiarrheal agents and acid inhibitors should be provided in those patients, so as to ensure the balance of intake and discharge.

The existing data supported that smoking history ${ }^{27,28}$, penetrating lesions ${ }^{27,29}$, postoperative perianal lesions ${ }^{28,29}$ and emergency surgery ${ }^{28}$ were risk factors for postoperative recurrence, whereas azathioprine ${ }^{27}$, antitumor necrosis factor ${ }^{29}$ and mesenteric resection ${ }^{30}$ were protective factors. Due to the long-term use of glucocorticoids, immunosuppressive agents and malnutrition in patients required surgical treatment, one-stage temporary ostomy to improve nutritional status, as well as two-stage ostomy and intestinal anastomosis were common modes of operation under these circumstances. However, there is no literature review about whether the recurrence rate of $C D$ patients is increased under staging operation compared with that of the one-stage definitive operation. In our study, there was no difference in the long-term reoperation rate between the staging operation and the one-stage definitive operation, and the operative complication was relatively low during intestinal anastomosis. Therefore, staging surgical program is recommended for patients with poor nutritional status, emergency operation and risk factors of postoperative intraperitoneal infectious complications.

There are some limitations to this study. Firstly, this study is a retrospective analysis of medical records, with certain bias of the data. Secondly, our Inflammatory Bowel Disease (IBD) Center is the only specialized IBD clinic in China, and the patients who are being transferred to our hospital have serious symptoms and complicated conditions. Thirdly, patients who underwent primary bowel resection in another hospital were excluded, leading to a small sample size. Nevertheless, this study is the first to explore the effect of residual lesions on reoperation rates after surgery for $\mathrm{CD}$. A prospective, large sample study is needed to validate the conclusions.

In conclusion, surgical intervention at proper time and appropriate operation during operation are essential for patients with CD. It is believed that staging operation with ostomy followed by intestinal anastomosis is feasible when there are more than two risk factors for postoperative intra-abdominal infectious complications. In addition, regular follow-up and medical treatment are of critical importance for CD patients. 


\section{References}

1. Lockhart-Mummery, H. E. M. Crohn's disease (regional enteritis) of the large intestine and its distinction from ulcerative colitis. Gut 1, 87-105 (1960).

2. Krupnick, A. S. M. The long-term results of resection and multiple resections in Crohn's disease. Seminars in gastrointestinal disease 11, 41-51 (2000).

3. Mekhjian, H. S. et al. National Cooperative Crohn's Disease Study: factors determining recurrence of Crohn's disease after surgery. Gastroenterology 7, 907-913 (1979).

4. Rotondo, M. F. S. et al. 'Damage control': an approach for improved survival in exsanguinating penetrating abdominal injury. The Journal of trauma 35, 375-382; discussion 382-373 (1993).

5. Gomollon, F. D. et al. European Evidence-based Consensus on the Diagnosis and Management of Crohn's Disease 2016: Part 1: Diagnosis and Medical Management. Journal of crohns \& colitis 11, 3-25 (2017).

6. Gionchetti, P. D. et al. European Evidence-based Consensus on the Diagnosis and Management of Crohn's Disease 2016: Part 2: Surgical Management and Special Situations. Journal of crohns \& colitis 11, 135-149 (2017).

7. Varadhan, K. N. et al. The enhanced recovery after surgery (ERAS) pathway for patients undergoing major elective open colorectal surgery: A meta-analysis of randomized controlled trials. Clinical nutrition 29, 434-440 (2010).

8. Gustafsson, U. S. et al. Guidelines for Perioperative Care in Elective Colonic Surgery: Enhanced Recovery After Surgery (ERAS((R))) Society Recommendations. World journal of surgery 37, 259-284 (2013).

9. Satsangi, J., Silverberg, M. S., Vermeire, S. \& Colombel, J. F. The Montreal classification of inflammatory bowel disease: controversies, consensus, and implications. GUT 55, 749-753 (2006).

10. Clavien, P. B. et al. The Clavien-Dindo Classification of Surgical Complications Five-Year Experience. ANNALS OF SURGERY 250, 187-196 (2009).

11. Issa, M. \& Binion, D. G. Bowel Rest and Nutrition Therapy in the Management of Active Crohn's Disease. Nutrition in clinical practice 23, 299-308 (2008).

12. Gutierrez, A. L. H. \& Sands, B. E. Outcome of surgical versus percutaneous drainage of abdominal and pelvic abscesses in Crohn's disease. American journal of gastroenterology 101, 2283-2289 (2006).

13. Xie, Y. Z. W. M., Li, N. \& Li, J. S. The outcome of initial percutaneous drainage versus surgical drainage for intra-abdominal abscesses in Crohn's disease. International journal of colorectal disease 27, 199-206 (2012).

14. Muller-Wille, R. I. et al. Influence of percutaneous abscess drainage on severe postoperative septic complications in patients with Crohn's disease. International journal of colorectal disease 26, 769-774 (2011).

15. Crowell, K. T. M. Evangelos. Risk factors and implications of anastomotic complications after surgery for Crohn's disease. World journal of gastrointestinal surgery 7, 237-242 (2015).

16. Iesalnieks, I. K. et al. Intraabdominal septic complications following bowel resection for Crohn's disease: detrimental influence on long-term outcome. International journal of colorectal disease 23, 1167-1174 (1167-1174).

17. Myrelid, P. S. J. D., Olaison, G., Sjodahl, R. \& Andersson, P. Split stoma in resectional surgery of high-risk patients with ileocolonic Crohn's disease. Colorectal disease 18, 188-193 (2012).

18. Fumery, M. S. et al. Postoperative Complications after Ileocecal Resection in Crohn's Disease: A Prospective Study From the REMIND Group. American journal of gastroenterology 112, 337-345 (2017).

19. Morar, P. H. et al. Determining Predictors for Intra-abdominal Septic Complications Following Ileocolonic Resection for Crohn's Disease-Considerations in Pre-operative and Peri-operative Optimisation Techniques to Improve Outcome. Journal of crohns \& colitis 9, 483-491 (2015).

20. Post, S. H. C., Schumacher, H., Golling, M., Schurmann, G. \& Timmermanns, G. Experience with ileostomy and colostomy in Crohn's disease. The British journal of surgery 82, 1629-1633 (1995).

21. McIlrath, D. C. Diverting ileostomy or colostomy in the management of Crohn's disease of the colon. Archives of surgery 103, 308-310 (1971)

22. Rutgeerts, P. G. et al. Effect of faecal stream diversion on recurrence of Crohn's disease in the neoterminal ileum. Lancet 338, 771-774 (1991).

23. Burman, J. H. T. H., Cooke, W. T. \& Williams, J. A. The effects of diversion of intestinal contents on the progress of Crohn's disease of the large bowel. Gut 12,11-15 (1971).

24. Cataldo, P. Intestinal stomas - 200 years of digging. Diseases of the colon \& rectum 42, 137-142 (1999).

25. Hirsch, A. Y. et al. Penetrating Disease, Narcotic Use, and Loop Ostomy Are Associated with Ostomy and IBD-related Complications After Ostomy Surgery in Crohn's Disease Patients. Journal of gastrointestinal surgery 19, 1852-1861 (2015).

26. Messaris, E. S. et al. Dehydration Is the Most Common Indication for Readmission After Diverting Ileostomy Creation. Diseases of the colon o rectum 55, 175-180 (2012).

27. Guo, J. C. et al. prevented surgical recurrence in patients with Crohn's disease after intestinal resection: Strategy based on risk stratification. Journal of gastroenterology and hepatology 33, 608-614 (2018).

28. Yang, K. Y. et al. Risk factors for postoperative recurrence after primary bowel resection in patients with Crohn's disease. World journal of gastroenterology 23, 7016-7024 (2017).

29. Kusaka, J. S. et al. Risk factors associated with postoperative recurrence and repeat surgery in Japanese patients with Crohn's disease. International journal of colorectal disease 32, 1407-1413 (2017).

30. Coffey, J. C. K. et al. Colum Inclusion of the mesentery in ileocolic resection for Crohn's disease is associated with reduced surgical recurrence. Journal of Crohn's \& colitis (2018).

\section{Author Contributions}

Jiajie Zhou, Yi Li and Jianfeng Gong participated in the study design, data collection, analysis of data and preparation of the manuscript. Weiming Zhu revised this paper critically for important intellectual content. Jiajie Zhou carried out the study design, the analysis and interpretation of data and drafted the manuscript. All authors read and approved the final manuscript.

\section{Additional Information}

Competing Interests: The authors declare no competing interests.

Publisher's note: Springer Nature remains neutral with regard to jurisdictional claims in published maps and institutional affiliations. 
(i) Open Access This article is licensed under a Creative Commons Attribution 4.0 International License, which permits use, sharing, adaptation, distribution and reproduction in any medium or format, as long as you give appropriate credit to the original author(s) and the source, provide a link to the Creative Commons license, and indicate if changes were made. The images or other third party material in this article are included in the article's Creative Commons license, unless indicated otherwise in a credit line to the material. If material is not included in the article's Creative Commons license and your intended use is not permitted by statutory regulation or exceeds the permitted use, you will need to obtain permission directly from the copyright holder. To view a copy of this license, visit http://creativecommons.org/licenses/by/4.0/.

(C) The Author(s) 2019 